David W. Desmond Thomas K. Tatemichi Miguel Figueroa Toby I. Gropen Yaakov Stern

\section{Disorientation following stroke: frequency, course, and clinical correlates}

Received: 1 December 1993

Received in revised form: 8 March 1994

Accepted: 15 March 1994

Presented in part

at the 118th Annual Meeting

of the American Neurological Association, Boston, Massachusetts, October 1993

D. W. Desmond , T. K. Tatemichi

M. Figueroa - T. I. Gropen - Y. Stern

Department of Neurology,

Columbia University,

College of Physicians and Surgeons,

New York, N.Y., USA

\section{Y. Stern}

Department of Psychiatry

and the Gertrude H. Sergievsky Center,

Columbia University,

College of Physicians and Surgeons,

New York, N.Y., USA

T. K. Tatemichi $(\bowtie)$

Neurological Institute,

710 West 168 th Street, New York,

NY 10032, USA
Abstract To investigate the frequency, course, and clinical correlates of disorientation following stroke, we administered the MiniMental State Examination orientation subtest to 177 alert patients $7-10$ days and 3 months after stroke and 240 stroke-free nondemented subjects. Disorientation was defined as a score $\leq 8 / 10$. Seventy-two $(40.7 \%)$ of the patients were disoriented 7-10 days after stroke and 39 patients (22.0\% of the sample) remained disoriented 3 months later. A logistic regression analysis determined that persistent disorientation was significantly related to stroke status [odds ratio $(O R)=5.8$, after adjusting for memory and attentional deficits and demographic variables. Among stroke patients, disorientation was associated with severe hemispheral stroke syndromes $(\mathrm{OR}=7.7)$, but not infarct location or vascular risk fac- tor history, after adjusting for memory and attentional deficits and demographic variables. Sensitivity and specificity analyses determined that disorientation was an inaccurate marker for dementia or deficits in memory or attention, while intact orientation was associated with a low probability of dementia or memory dysfunction in most patients but not preserved attention. We conclude that disorientation is common and persistent following stroke and associated with severe hemispheral stroke syndromes but not infarct location. While disorientation is a poor marker for dementia or deficits in memory or attention, intact orientation should suggest that cognitive functions are likely to be preserved.

Key words Disorientation - Stroke Dementia $\cdot$ Memory $\cdot$ Attention

\section{Introduction}

Disorientation, a state of confusion regarding time, location, or identity, is considered to be a marker for dementia by many clinicians. A number of studies have reported an association between disorientation and diffuse neurologic disorders that may lead to dementia, including Alzheimer's disease $[10,11,14]$, Parkinson's disease [10], closed head injury [7, 13], Korsakoff's syndrome [12], and Down's syndrome [18]. Disorientation has rarely been a primary focus of study, however, and it is usually discussed as one of many potential deficits in general cognitive perfor- mance. Although orientation may be the most easily and frequently assessed cognitive function in bedside examinations, it is also the least understood.

The primary aim of this study was to investigate the clinical correlates of persistent disorientation following stroke, with a specific focus upon infarct location, presenting stroke syndrome, and history of exposure to risk factors for cerebrovascular disease. Our primary hypothesis was that disorientation would be associated with more severe stroke syndromes, but not specific stroke locations. The secondary aim of this study was to examine the relationship between orientation and other cognitive functions in the context of a neuropsychological test battery to de- 
termine whether disorientation can be considered to be a valid marker for dementia or deficits in specific cognitive domains, such as memory and attention. Our secondary hypothesis was that disorientation would be a poor marker for dementia or deficits in specific cognitive functions.

\section{Subjects and methods}

As part of a prospective study of stroke and dementia [17], we examined 177 patients (age $=70.6$, SD 7.9 years; education $=10.2, \mathrm{SD}$ 4.8 years) both $7-10$ days and 3 months after stroke who were admitted to Columbia-Presbyterian Medical Center. Those patients were selected from a larger cohort of 297 patients, with the remaining patients having been excluded because they were not assessed with the Mini-Mental State Examination (MMSE) [4] at both of those intervals $(n=103)$ and/or because they had received a diagnosis of possible Alzheimer's disease with stroke based on a premorbid history of functional impairment and neuropsychological testing consistent with dementia $(n=24)$. All stroke patients included in this study were found to be alert on neurologic examination.

To investigate the likelihood of disorientation after stroke and define a cutoff for disorientation based upon MMSE performance, 240 stroke-free nondemented control subjects (age $=70.6$, SD 6.5 years; education $=12.5$, SD 4.4 years) were also examined. The majority of these subjects were randomly selected from Medicare records and a commercial list $(52.1 \%)$, with the remainder of the cohort being composed of volunteers from the community $(30.8 \%)$ and spouses of stroke patients $(\mathbf{1 7 . 1 \% )}$ ). Because we were attempting to investigate persistent disorientation in stable stroke patients rather than the acute effects of hospitalization, we felt that it was appropriate to examine an outpatient control sample rather than acutely hospitalized patients free of neurologic disorder.

\section{Test instruments}

Cognitive testing was performed in either English or Spanish, based upon the language spoken in the subject's home. The following test instruments were used:

\section{MMSE, orientation subtest}

This subtest [4] includes ten questions assessing orientation to time (i.e., month, date, year, day of week, season) and location (i.e., city, state, borough, name of hospital, floor). Given that the MMSE was administered in a standard fashion, orientation to personal identity was not assessed. Disorientation was defined as a score of $\leq 8 / 10$ on this subtest, this cutoff falling 3 SD below the mean for the control sample (i.e., <1st percentile). Persistent disorientation was defined as MMSE orientation subtest scores falling below this cutoff at both $7-10$ days and 3 months after stroke. Test-retest reliability is high for the MMSE as a whole [4].

\section{Memory and attentional tasks}

To investigate the association between disorientation and memory and attentional dysfunction, we administered the Selective Reminding Test as a measure of verbal memory, a multiple-choice recognition version of the Benton Visual Retention Test as a measure of nonverbal memory, and a timed graphomotor target cancellation task as a measure of attention. These tests are sensitive to many but not all aspects of memory and attentional dysfunction. They were taken from a comprehensive neuropsychological test battery [16] developed for use in epidemiologic studies of dementia.

The MMSE orientation subtest was administered to all patients 7-10 days after stroke by trained research assistants and it was readministered in conjunction with the memory and attentional tasks 3 months after stroke.

\section{Additional procedures}

Neurologists specializing in stroke (T.K.T, M.F., T.I.G.) administered a structured interview to all subjects regarding history of stroke, transient ischemic attack, and exposure to risk factors for cerebrovascular disease. We have found in a previous study that subject self-reports regarding risk factor history are both reliable and valid [3]

Based upon the review of clinical features and CT scans performed immediately after stroke, patients were classified by severity of clinical syndrome, infarct location, and vascular territory of infarction using a modification of the methods of the Stroke Data Bank [5]. Presenting stroke syndrome was classified as major hemispheral or other, reflecting the severity of neurologic impairment. A patient with a transcortical motor aphasia, ideomotor dyspraxia, and severe right hemiparesis with crural predominance, for example, would be classified as having a major hemispheral syndrome, with widespread damage in the anterior cerebral artery territory of the dominant hemisphere. A patient with anosognosia, left hemi-inattention and hemianopia, and left facial-brachial paresis would be considered to have a major hemispheral syndrome due to infarction in the middle cerebral artery territory of the nondominant hemisphere. Infarct location was classified as left hemisphere, frontal; left hemisphere, nonfrontal; right hemisphere, frontal; right hemisphere, nonfrontal; bilateral, with a frontal component; bilateral, without a frontal component; or brainstem/cerebellar. Vascular territory of infarction was classified as anterior cerebral artery, middle cerebral artery, posterior cerebral artery, internal carotid artery (i.e., borderzone territories), the deep penetrating component of any of the preceding arteries, or vertebrobasilar system. Classifications by stroke syndrome, location, and vascular territory were independent of information on neuropsychological testing.

\section{Statistical analyses}

To determine whether any selection bias might have resulted from the exclusion of a large proportion of the stroke patients in the original cohort, univariate analyses (i.e., $t$-tests for continuous data and chi-square analyses for categorical data throughout) were performed to compare the patients who were excluded from this study to those patients who were included with regard to demographic variables and the results of neurologic, functional, and mental status examinations performed 7-10 days after stroke. When data from the 7-10 day interval were not available, the results of examinations performed 30 days after stroke were used. We then performed a logistic regression analysis to determine whether any of the variables found to be significant in the univariate analyses would be independently related to exclusion from this study. $\mathrm{Pa}$ tients excluded due to a diagnosis of possible Alzheimer's disease with stroke were not relevant for these analyses.

To investigate the likelihood of persistent disorientation after stroke, we performed a logistic regression analysis, with presence or absence of persistent disorientation as the dependent variable and stroke status as the independent variable. Disorientation was defined as an MMSE orientation subtest score of $\leq 8 / 10$ at both 7-10 days and 3 months after stroke for the stroke sample and at baseline for the control sample. Demographic variables were forced into the final model to adjust for their effects. To understand the relationship between disorientation and memory and at- 
tentional dysfunction better, we performed this and each of the subsequent analyses twice, first without dichotomized variables representing memory and attentional dysfunction and then with those variables. To define these variables, we transformed scores on the memory and attentional tasks into $\mathrm{z}$-scores based upon normative means and standard deviations derived from the performance of the control sample. Scores more than 2SD below the mean for the control sample (i.e., approximately the 2nd percentile) were considered defective for each of these cognitive domains. A cutoff of 2 SD below the mean was used rather than 3 SD because the latter criterion would have identified too small a number of patients with defective memory to permit reliable statistical analyses.

To investigate the clinical correlates of generalized persistent disorientation after stroke, we first performed chi-square analyses based upon the stroke sample. The independent variable in each of these analyses was the presence or absence of persistent disorientation and the dependent variables included presenting stroke syndrome, infarct location, vascular territory of infarction, history of prior stroke, history of transient ischemic attack, history of exposure to specific risk factors for cerebrovascular disease, location on admission (i.e., intensive care or step-down unit versus general ward), medical complications during hospitalization (e.g., cardiac or respiratory arrest, pneumonia, hyponatremia), medication use, location 3 months after stroke (i.e., home versus any other location), and demographic factors. We then performed a logistic regression analysis, with presence or absence of persistent disorientation as the dependent variable and variables found to be significantly related to persistent disorientation in the univariate analyses as independent variables. Demographic variables were forced into the final model to adjust for their effects. These analyses were then repeated with the memory and attention variables being added.

We also examined the value of disorientation as a marker for dementia, defective memory, and defective attention. Defective memory and attention were defined as described above. Dementia was diagnosed using a standardized paradigm based upon neuropsychological testing conducted 3 months after stroke [16]. Sensitivity, specificity, positive predictive value, and negative predictive value were calculated. To ensure that the results of these analyses were not biased by our transformation of continuous data into dichotomous variables, we also calculated correlation coefficients representing the association between MMSE orientation subtest score and each of the other variables, with the results of the cognitive tests administered 3 months after stroke being treated as continuous data and dementia status remaining a dichotomous variable.

\section{Results}

\section{Potential selection biases}

Univariate analyses determined that the group of patients who had been excluded from this study were more often demented, as indicated by an MMSE total score <24; were more often aphasic; had experienced a more severe stroke, as indicated by a higher Stroke Severity score; were more physically disabled, as indicated by a lower Barthel Index score; and had a history of greater functional impairment prior to stroke onset, as indicated by a higher Blessed Functional Activity Scale score, than those who were included in this study. These results are presented in Table 1. A logistic regression analysis determined that aphasia and a lower Barthel Index score were significant independent correlates of exclusion from this study.
Table 1 Characteristics of included and excluded subjects. Figures are frequencies and within-group percentages unless otherwise noted. Significance levels are based on $t$-tests for continuous data and chi-square analyses for frequency data. Test data are taken from examinations conducted 7-10 days and, when necessary, 30 days after stroke. Some within-group percentages are based on incomplete sample of excluded subjects due to untestability (MMSE Mini-Mental State Examination, BFAS Blessed Functional Activity Scale)

\begin{tabular}{lllr}
\hline Variable & $\begin{array}{l}\text { Included } \\
(n=177)\end{array}$ & $\begin{array}{l}\text { Excluded } \\
(n=96)\end{array}$ & $P$ \\
\hline Age (mean, SD) & $70.6,7.9$ & $72.2,7.8$ & 0.104 \\
Education (mean, SD) & $10.2,4.8$ & $9.9,4.5$ & 0.586 \\
Race (nonwhite) & $123(69.5)$ & $57(59.4)$ & 0.092 \\
Sex (male) & $86(48.6)$ & $40(41.7)$ & 0.273 \\
MMSE total score (mean, SD) & $23.4,5.1$ & $19.9,7.5$ & $<0.001$ \\
MMSE total score < 24 & $75(42.4)$ & $48(59.3)$ & 0.012 \\
MMSE orientation score & $8.3,2.0$ & $7.2,2.9$ & $<0.001$ \\
$\quad$ (mean, SD) & & & \\
MMSE orientation score $\leq 8$ & $72(40.7)$ & $42(56.0)$ & 0.026 \\
Aphasia & $21(12.5)$ & $24(27.9)$ & 0.002 \\
Stroke Severity (mean, SD) & $6.2,3.2$ & $7.1,3.3$ & 0.038 \\
Barthel Index (mean, SD) & $70.5,26.6$ & $62.8,32.3$ & 0.039 \\
BFAS score (mean, SD) & $0.6,1.5$ & $1.0,2.3$ & 0.039 \\
\hline
\end{tabular}

MMSE orientation subtest scores were not included in the preceding analyses. Rather, MMSE total score was used in order to diagnose dementia, which would seem to be a more likely correlate of exclusion from this study than isolated disorientation. As shown in Table 1, however, patients who were excluded from this study received significantly lower scores on the MMSE orientation subtest administered 7-10 days or 30 days after stroke than the patients who were included.

Risk, frequency, and persistence of disorientation after stroke

Univariate analyses determined that stroke status, older age, and nonwhite race were significantly related to disorientation. A logistic regression analysis, with disorientation as the dependent variable and stroke status and demographic factors as independent variables, determined that stroke patients were at significantly increased risk of disorientation relative to control subjects [odds ratio (OR) $=10.9 ; 95 \%$ confidence interval $(\mathrm{CI})=4.0-29.4]$, while adjusting for the significant effects of age $(\mathrm{OR}=3.0 ; \mathrm{CI}=$ $1.1-8.6$ for $80+$ versus $60-69$ and $\mathrm{OR}=2.3 ; \mathrm{CI}=1.1-5.1$ for $70-79$ versus $60-69)$ and race $(\mathrm{OR}=3.2 ; \mathrm{CI}=1.3-8.2$, nonwhites worse) and the nonsignificant effects of education and sex. After entering variables representing performance in memory and attentional testing, a further logistic regression analysis determined that stroke patients remained at significantly increased risk of disorientation relative to control subjects ( $\mathrm{OR}=5.8 ; \mathrm{CI}=2.0-16.6)$, while 
Table 2 Demographic variables and neurologic and medical history by presence or absence of persistent disorientation. Figures are frequencies and within-group percentages. Significance levels are based on chi-square analyses

\begin{tabular}{|c|c|c|c|}
\hline \multirow[t]{2}{*}{ Variable } & \multicolumn{3}{|c|}{ Persistent disorientation } \\
\hline & $\begin{array}{l}\text { Yes } \\
(n=39)\end{array}$ & $\begin{array}{l}\text { No } \\
(n=138)\end{array}$ & $P$ \\
\hline \multicolumn{4}{|l|}{ Age } \\
\hline $60-69$ years old & $14(35.9)$ & $77(55.8)$ & 0.047 \\
\hline $70-79$ years old & $17(43.6)$ & $48(34.8)$ & \\
\hline $80+$ years old & $8(20.5)$ & $13 \quad(9.4)$ & \\
\hline Education ( $<13$ years) & $34(87.2)$ & $99(71.7)$ & 0.049 \\
\hline Race (nonwhile) & $34(87.2)$ & $89(64.5)$ & 0.007 \\
\hline Sex (male) & $16(41.0)$ & $70(50.7)$ & 0.295 \\
\hline Prior stroke & $10(25.6)$ & $28(20.3)$ & 0.472 \\
\hline Prior transient ischemic attack & $4(10.5)$ & $29(21.2)$ & 0.138 \\
\hline Hypertension & $29(74.4)$ & $100(72.5)$ & 0.814 \\
\hline Diabetes mellitus & $20(51.3)$ & $44(31.9)$ & 0.026 \\
\hline Myocardial infarction & $3 \quad(7.9)$ & $27(19.6)$ & 0.090 \\
\hline Congestive heart failure & $5(12.8)$ & $16(11.8)$ & 0.858 \\
\hline Valvular heart disease & $1 \quad(2.6)$ & $8 \quad(5.9)$ & 0.408 \\
\hline Angina & $7(18.4)$ & $32(23.4)$ & 0.518 \\
\hline Atrial fibrillation & $8(20.5)$ & $20(14.6)$ & 0.373 \\
\hline Consistent cigarette use & $21(53.8)$ & $88(63.8)$ & 0.261 \\
\hline Consistent alcohol use & $11(28.2)$ & $75(54.3)$ & 0.004 \\
\hline
\end{tabular}

adjusting for the significant effects of defective memory $(\mathrm{OR}=5.7 ; \mathrm{CI}=1.9-17.0)$ and race $(\mathrm{OR}=2.8 ; \mathrm{CI}=1.0-8.0$, nonwhites worse), the weak effects of defective attention $(\mathrm{OR}=2.2 ; \mathrm{CI}=0.9-5.3)$, and the nonsignificant effects of age, education, and sex. Relative to the cutoff for disorientation based upon control group performance, 72 $(40.7 \%)$ of the patients were disoriented 7-10 days after stroke and 39 of those patients (22.0\% of the sample) remained disoriented 3 months later.

\section{Clinical correlates of disorientation after stroke}

Descriptive statistics and the results of the chi-square analyses are presented in Tables 2-4. Variables significantly related to persistent disorientation in these analyses were: a major stroke syndrome; infarcts in a number of hemispheral locations; vascular territory of infarction, particularly in the anterior and posterior cerebral artery territories; a history of diabetes mellitus; a history of consistent alcohol use; medical complications during hospitalization; older age; fewer years of education; and nonwhite race.

A logistic regression analysis determined that persistent disorientation was significantly related to infarctions in the anterior cerebral artery $(\mathrm{OR}=15.1 ; \mathrm{Cl}=1.5-148.1)$, middle cerebral artery $(\mathrm{OR}=5.7 ; \mathrm{CI}=1.1-29.1)$, posterior cerebral artery $(\mathrm{OR}=25.2 ; \mathrm{CI}=2.9-222.2)$ and deep pen-
Table 3 Infarct characteristics by presence or absence of persistent disorientation. Figures are frequencies and within-group percentages. Significance levels are based on chi-square analyses

\begin{tabular}{|c|c|c|c|}
\hline \multirow[t]{2}{*}{ Variable } & \multicolumn{3}{|c|}{ Persistent disorientation } \\
\hline & $\begin{array}{l}\text { Yes } \\
(n=39)\end{array}$ & $\begin{array}{l}\text { No } \\
(n=138)\end{array}$ & $P$ \\
\hline Major stroke syndrome & $15(38.5)$ & $14(10.1)$ & $<0.001$ \\
\hline Aphasia & $6(17.1)$ & $17(12.8)$ & 0.504 \\
\hline $\begin{array}{l}\text { Infarct location } \\
\text { Left hemisphere, frontal } \\
\text { Left hemisphere, nonfrontal } \\
\text { Right hemisphere, frontal } \\
\text { Right hemisphere, nonfrontal } \\
\text { Bilateral, frontal component } \\
\text { Bilateral, no frontal component } \\
\text { Brainstem/cerebellum }\end{array}$ & $\begin{array}{rr}6 & (15.4) \\
10 & (25.6) \\
2 & (5.1) \\
9 & (23.1) \\
4 & (10.3) \\
7 & (17.9) \\
1 & (2.6)\end{array}$ & $\begin{array}{rr}10 & (7.2) \\
20 & (14.5) \\
4 & (2.9) \\
24 & (17.4) \\
8 & (5.8) \\
24 & (17.4) \\
48 & (34.8)\end{array}$ & 0.007 \\
\hline $\begin{array}{l}\text { Vascular territory } \\
\text { Anterior cerebral artery } \\
\text { Middle cerebral artery } \\
\text { Posterior cerebral artery } \\
\text { Internal carotid artery } \\
\text { Deep penetrating arteries } \\
\text { Vertebrobasilar artery }\end{array}$ & $\begin{array}{rr}4 & (10.3) \\
15 & (38.5) \\
5 & (12.8) \\
1 & (2.6) \\
11 & (28.2) \\
3 & (7.7\}\end{array}$ & $\begin{array}{rr}4 & (2.9) \\
46 & (33.3) \\
7 & (5.1) \\
4 & (2.9) \\
29 & (21.0) \\
48 & (34.8)\end{array}$ & 0.010 \\
\hline
\end{tabular}

etrating artery $(\mathrm{OR}=14.1 ; \mathrm{CI}=2.6-76.8)$ territories relative to the vertebrobasilar system, while infarctions in the internal carotid distribution (i.e., borderzone territories) were unrelated; a major hemispheral syndrome, reflecting the severity of the stroke (OR=4.6; $\mathrm{CI}=1.4-15.0)$; and medical complications during hospitalization $(\mathrm{OR}=3.4$; $\mathrm{CI}=1.1-10.2)$; and weakly related to a history of diabetes mellitus ( $\mathrm{OR}=2.5 ; \mathrm{CI}=1.0-6.4)$; while adjusting for the significant effects of age $(\mathrm{OR}=12.9 ; \mathrm{CI}=2.8-58.8$ for $80+$ versus $60-69$ and $\mathrm{OR}=4.2 ; \mathrm{CI}=1.3-13.4$ for $70-79$ versus $60-69)$ and race $(\mathrm{OR}=5.5 ; \mathrm{CI}=1.4-21.4$, nonwhites worse) and the nonsignificant effects of education and sex. Because the odds ratios related to anterior and posterior cerebral artery territory infarctions appeared to be particularly high, the logistic regression analysis was repeated, with the anterior and posterior cerebral artery territories being pooled and compared with all other territories, this variable essentially representing infarctions in the territories that supply the limbic system versus all other territories. This variable was significantly related to persistent disorientation in the final model $(\mathrm{OR}=6.0 ; \mathrm{CI}=$ 2.0-17.9), and all of the other variables included in the final model in the preceding analysis also remained in this model.

We repeated the original logistic regression analysis, adding dichotomous variables representing performance in memory and attentional testing, and determined that a major stroke syndrome $(\mathrm{OR}=7.7 ; \mathrm{CI}=2.6-22.9)$ was the only significant clinical correlate from the preceding 
Table 4 Course of hospitalization by presence or absence of persistent disorientation. Figures are frequencies and within-group percentages. Significance levels are based on chi-square analyses. ( $N V U / / C U$ neurovascular unit or intensive care unit)

\begin{tabular}{|c|c|c|c|}
\hline \multirow[t]{2}{*}{ Variable } & \multicolumn{3}{|c|}{ Persistent disorientation } \\
\hline & $\begin{array}{l}\text { Yes } \\
(n=39)\end{array}$ & $\begin{array}{l}\text { No } \\
(n=138)\end{array}$ & $P$ \\
\hline Admission location (NVU/ICU) & $28(71.8)$ & $85(61.6)$ & 0.242 \\
\hline \multicolumn{4}{|l|}{ Medication use } \\
\hline Antihypertensives & $15(38.5)$ & $68(49.3)$ & 0.232 \\
\hline Insulin/oral hypoglycemics & $14(35.9)$ & $29(21.0)$ & 0.056 \\
\hline Cardiac medications & $21(53.8)$ & $65(47.1)$ & 0.457 \\
\hline Antihyperlipidemics & $0 \quad(0.0)$ & $3 \quad(2.2)$ & 0.353 \\
\hline Anxiolytics/sedatives & $1 \quad(2.6)$ & $18(13.0)$ & 0.062 \\
\hline Anticonvulsants & $2 \quad(5.1)$ & $5 \quad(3.6)$ & 0.670 \\
\hline Antipsychotics/antidepressants & $2 \quad(5.1)$ & $8 \quad(5.8)$ & 0.873 \\
\hline Other medications & $37(94.9)$ & $117(84.8)$ & 0.098 \\
\hline Medical complications & $12(30.8)$ & $20(14.6)$ & 0.021 \\
\hline Location at 3 months (home) & $33(84.6)$ & $129(93.5)$ & 0.079 \\
\hline
\end{tabular}

analysis to remain in this model, while adjusting for the significant effects of defective memory $(\mathrm{OR}=5.7 ; \mathrm{CI}=$ 1.7-19.2) and race $(\mathrm{OR}=5.6 ; \mathrm{CI}=1.3-24.4$, nonwhites worse) and the nonsignificant effects of age, education, and sex. Infarct location and vascular territory of infarction, diabetes mellitus, medical complications during hospitalization, and defective attention were not significantly related to persistent disorientation in this final model.

Although it was not a primary focus of this study, we also performed this analysis using the presence or absence of disorientation 7-10 days after stroke as the dependent variable. The result was comparable, with a major stroke syndrome found to be the only significant clinical correlate of disorientation immediately after stroke, while adjusting for the significant effects of defective memory and attention as well as demographic variables.

Two questions could be posed regarding the role of infarct location in the preceding analyses. First, could the basis for the lack of a significant association between infarct location and disorientation in each of these analyses have been that our definition of infarct location was too specific (e.g., "left hemisphere infarction with a frontal component" rather than "left hemisphere infarction")? Second, did the use of patients with brainstem/cerebellar infarctions as a reference group compromise our ability to recognize significant differences in the frequency of disorientation among groups of patients with infarcts in different hemispheral locations? To address these questions, we performed additional univariate analyses in which we compared patients with left hemisphere, right hemisphere, and bilateral infarcts with regard to persistent generalized disorientation, these analyses excluding those patients
Table 5 Persistent disorientation as a marker for dementia, defective memory, and defective attention. Values are presented with $95 \%$ confidence intervals

\begin{tabular}{lllll}
\hline $\begin{array}{l}\text { Predicted } \\
\text { outcome }\end{array}$ & Sensitivity & Specificity & $\begin{array}{l}\text { Positive } \\
\text { predictive } \\
\text { value }\end{array}$ & $\begin{array}{l}\text { Negative } \\
\text { predictive } \\
\text { value }\end{array}$ \\
\hline $\begin{array}{c}\text { Dementia } \\
\text { Defective } \\
\text { memory }\end{array}$ & $0.59 \pm 0.19$ & $0.85 \pm 0.06$ & $0.41 \pm 0.15$ & $0.92 \pm 0.05$ \\
$\begin{array}{c}\text { Defective } \\
\text { attention }\end{array}$ & $0.34 \pm 0.11$ & $0.91 \pm 0.06$ & $0.74 \pm 0.15$ & $0.65 \pm 0.08$ \\
\hline
\end{tabular}

with brainstem/cerebellar infarctions. Significant differences among these groups were not obtained, suggesting that the univariate associations that were recognized between infarct location and disorientation when the brainstem/cerebellar group was included were the result of infarctions throughout the left and right hemispheres producing disorientation in a significantly greater proportion of patients than brainstem/cerebellar infarctions rather than any differences among specific hemispheral infarct locations.

Disorientation as a marker for other cognitive deficits

Finally, we considered disorientation as a correlate of dementia status as well as dysfunction in the domains of memory and attention. The results of these analyses are presented in Table 5, and they suggest that the sensitivity of disorientation is moderate as a marker for dementia and memory dysfunction and low as a marker for attentional dysfunction, while the specificity of disorientation is high for all of these disorders. The positive predictive value of disorientation is low for dementia and memory dysfunction and relatively high for attentional dysfunction, while the negative predictive value is high for dementia and memory dysfunction and moderate for attentional dysfunction.

When these associations were reexamined using the results of cognitive testing performed at the 3-month examination as continuous data, the correlations between MMSE orientation subtest score and memory $(r=0.52$, $P<0.001)$ and attention $(r=0.43, P<0.001)$ scores were moderate, although significant. The correlation between MMSE orientation subtest score and dementia status, again used as a dichotomous variable, was also moderate and significant $(r=-0.41, P<0.001)$.

\section{Discussion}

Our results suggest that disorientation is common and persistent after stroke and may be associated with severe hemispheral stroke syndromes, but not infarct location or 
lateralization, vascular territory of infarction, aphasia, prior stroke or transient ischemic attack, or exposure to risk factors for cerebrovascular disease, after adjusting for memory and attentional deficits, factors related to hospitalization, and demographic characteristics. Given that the patients who were excluded from this study and who were clinically free of Alzheimer's disease received significantly lower scores on the MMSE orientation subtest and exhibited more severe stroke syndromes and a greater frequency of aphasia than the patients who were included in this study, however, it is likely that the frequency of disorientation after stroke and the contribution of left hemisphere lesions to disorientation were underestimated.

Few other studies have focused specifically upon disorientation resulting from stroke. We have reported previously that a grouping of deficits in orientation, memory, and attention best distinguished between patients with first stroke and stroke-free nondemented elderly subjects with regard to cognitive function, suggesting that the cognitive deficits produced by stroke were greatest in those domains [2]. Wade et al. [20] found that $14 \%$ of a cohort of stroke patients were disoriented in assessments performed both 0-7 days and 6 months after stroke; a large proportion of the remaining patients were considered to be unassessable, however, suggesting that the frequency of disorientation after stroke may have been underestimated. Similar to our findings, disorientation immediately after stroke was associated with more severe stroke syndromes in that study, but not with infarct lateralization, as inferred from the side of weakness.

A small number of studies have investigated the relationship between other forms of brain damage and disorientation in heterogeneous patient samples. One study found that bilateral lesions affecting the frontal lobes were associated with significantly greater disorientation than lesions involving either the left or right frontal lobe [1]. Some studies using electroconvulsive therapy have suggested that bilateral electrode placement produces greater disorientation than unilateral placement [19], while other studies have found that disorientation is greatest with left hemisphere placement $[6,8]$. It has been suggested that closed head injury with frontal impact may produce more persistent disorientation than impact involving other sites [9].

Our preliminary model of the clinical correlates of persistent disorientation after stroke suggested that orientation may be compromised by infarctions in a number of vascular territories, implicating many regions of the brain in the performance of this function. Infarctions in the territories of the anterior, middle, and posterior cerebral arteries, excluding those that resulted from penetrating artery occlusion, were all found to be associated with disorientation relative to infarctions in the territory of the vertebrobasilar system, suggesting that damage to the cortical regions supplied by these arterial systems may produce disorientation. Infarctions in the territories of the deep penetrating arteries were also found to be independently related, suggesting that subcortical structures may also play a role in maintaining orientation. Although infarctions in each of these vascular territories were significantly related to disorientation, the odds ratios resulting from the logistic regression analysis suggested that infarctions in the distributions of the anterior and posterior cerebral artery territories placed patients at approximately 3 times the risk of infarctions in the distribution of the middle cerebral artery (i.e., 13.2 and 14.7 versus 4.7 ). When we pooled the anterior and posterior cerebral artery territories in the logistic regression analysis, we found that infarctions in these territories were significantly related to persistent disorientation when compared to all other territories.

These arterial systems supply many of the structures in the limbic system, such as the medial frontal lobe and the inferomedial temporal lobe, which are understood to play an important role in memory and attentional functions [15]. When we repeated our logistic regression analysis and added variables representing dysfunction in the domains of memory and attention, we found that the only significant clinical correlate to remain in the final model was a major hemispheral stroke syndrome, suggesting that our initial finding of a relationship between infarcts involving the limbic system and disorientation was more likely a function of the role of the limbic system in mediating memory and attentional skills. That is, limbic system infarctions most likely produced primary disorders of memory and/or attention, which compromised the maintenance of orientation.

The results of our analyses regarding the value of disorientation as a marker for dysfunction within other cognitive domains have important implications for clinical practice. We found that the sensitivity of disorientation as a marker for dementia and deficits in memory and attention is moderate to low while specificity is high. These results suggest that the clinician should not assume that patients presenting with disorientation are demented, amnestic, or inattentive, while patients presenting with intact orientation can be expected to be free of dementia or memory dysfunction in many cases. Although it should be noted that the predictive value of a test is, in part, a function of the base rate of a disorder, our results also suggest that the clinician should not assume that patients who are oriented have intact attentional skills due to the moderate negative predictive value associated with this characteristic. To ensure that cognitive functions are characterized accurately, a comprehensive neuropsychological assessment should be performed.

Our study has certain limitations. First, we examined a control group composed of stroke-free volunteers from the community rather than hospitalized patients free of neurologic disorders. Although the use of this group allowed us to make an accurate determination of the frequency of disorientation 3 months after stroke, when over 
$90 \%$ of the stroke sample was living at home, it may have resulted in an overestimation of the frequency of disorientation 7-10 days after stroke. Second, we recognize that we have performed many analyses without having made Bonferroni adjustments to minimize the risk of type I error. We felt that the use of a Bonferroni adjustment would have resulted in a criterion for significance that would have been unreasonably strict, thus rendering it impossible for us to recognize any potentially subtle effects of the variables that we were exploring. Third, it is possible that we failed to recognize an association between stroke location and disorientation because we relied solely on structural brain imaging. The use of positron emission tomography or single photon emission computed tomography would have allowed us to investigate the role of strategically placed infarcts in the production of disorientation through the mechanism of disordered brain metabolism, which may involve sites remote from the region of infarction that is identified with structural brain imaging. Fourth, we did not investigate disorientation to personal identity in this study. Although this is a disorder rarely encountered in neurologic practice, future studies should attempt to investigate its clinical correlates.

Despite these limitations, our results suggest that measures of orientation may be quite sensitive to the effects of stroke and useful in the assessment of recovery. Disorientation following stroke might suggest a more severe hemispheral infarction, but not infarct location or lateralization, vascular territory of infarction, aphasia, prior stroke or transient ischemic attack, or exposure to specific risk factors for cerebrovascular disease. Disorientation may exist in association with disorders of memory or attention, but patients presenting with disorientation should not be assumed to be demented, amnestic or inattentive. Many patients who present with intact orientation, however, can be expected to be free of dementia or memory dysfunction.

This work was supported in part by grants R01-NS26179 and P01AG07232 and Clinical Investigator Development Award K08NS01635 (Dr. Desmond) from the National Institutes of Health.

\section{References}

1. Benton AL (1968) Differential behavioral effects in frontal lobe disease. Neuropsychologia 6:53-60

2. Desmond DW, Tatemichi TK, Stern Y, Sano M (1992) Cognitive dysfunction following first stroke (abstract). Neurology 42 [Suppl 3]:426

3. Desmond DW, Tatemichi TK, Paik M, Stern Y (1993) Risk factors for cerebrovascular disease as correlates of cognitive function in a stroke-free cohort. Arch Neurol 50:162-166

4. Folstein MF, Folstein SE, McHugh PR (1975) "Mini-Mental State": a practical method for grading the cognitive state of patients for the clinician. J Psychiatr Res 12:189-198

5. Foulkes MA, Wolf PA, Price TR, Mohr JP, Hier DB (1988) The Stroke Data Bank: design, methods, and baseline characteristics. Stroke 19:547-554

6. Gottlieb G, Wilson I (1965) Cerebral dominance: temporary disruption of verbal memory by unilateral electroconvulsive shock treatment. J Comp Physiol Psychol 60:368-372

7. Groher M (1977) Language and memory disorders following closed head in jury. J Speech Hear Res 20:212-223

8. Halliday AM, Davison K, Browne MW, Kreeger LC (1968) A comparison of the effects on depression and memory of bilateral E.C.T. and unilateral E.C.T. to the dominant and nondominant hemispheres. Br J Psychiatry 114:997-1012
9. High WM, Levin HS, Gary HE (1990) Recovery of orientation following closed-head injury. J Clin Exp Neuropsychol 12:703-714

10. Huber SI, Shuttleworth EC, Freidenberg DL (1989) Neuropsychological differences between the dementias of Alzheimer's and Parkinson's diseases. Arch Neurol 46:1287-1291

11. Hunter R, McLuskie R, Wyper D, Patterson J, Christie JE, Brooks DN, McCulloch J, Fink G, Goodwin GM (1989) The pattern of function-related regional cerebral blood flow investigated by single photon emission tomography with ${ }^{99 m} \mathrm{Tc}-\mathrm{HMPAO}$ in patients with presenile Alzheimer's disease and Korsakoff's psychosis. Psychol Med 19:847-855

12. Kopelman MD (1986) Clinical tests of memory. Br J Psychiatry 148:517-525

13. Levin HS, Grossman RG (1978) Behavioral sequelae of closed head injury: a quantitative study. Arch Neurol 35:720-727

14. Mahurin RK, DeBettignies BH, Pirozzolo FJ (1991) Structured Assessment of Independent Living Skills: preliminary report of a performance measure of functional abilities in dementia. J Gerontol 46:P58-P66

15. Mesulam MM (1990) Large-scale neurocognitive networks and distributed processing for attention, language, and memory. Ann Neurol 28:597-613
16. Stern Y, Andrews H, Pittman J, Sano M. Tatemichi T, Lantigua $R$, Mayeux $R$ (1992) Diagnosis of dementia in a heterogeneous population: development of a neuropsychological paradigm-based diagnosis of dementia and quantified correction for the effects of education. Arch Neurol 49:453-460

17. Tatemichi TK, Desmond DW, Mayeux R, Paik M, Stern Y, Sano M, Remien RH, Williams JBW, Mohr JP, Hauser WA, Figueroa M (1992) Dementia after stroke: baseline frequency, risks, and clinical features in a hospitalized cohort. Neurology 42:1185-1 193

18. Thase ME, Liss L, Smeltzer D, Maloon $\mathrm{J}$ (1982) Clinical evaluation of dementia in Down's syndrome: a preliminary report. J Ment Defic Res 26:239-244

19. Valentine M, Keddie KMG, Dunne D (1968) A comparison of techniques in electro-convulsive therapy. Br J Psychiatry 114:989-996

20. Wade DT, Skilbeck C, Hewer RL (1989) Selected cognitive losses after stroke: frequency, recovery, and prognostic importance. Int Disabil Stud $11: 34-39$ 